\title{
Fear Versus Scare Appeals as Moderators in Effective Health Messaging
}

\author{
Christopher D. Hopkins, Kevin J. Shanahan, Karen Hood, and Allyn White
}

\begin{abstract}
Fear appeals are often engaged in prosocial change marketing campaigns with the belief that these messages will raise severity and susceptibility beliefs among consumers. With effective advertising messages, persuasion models from the marketing literature suggest that if consumers believe the advertising claims, have positive attitudes toward the advertisements, that will produce both information seeking and medical care seeking behaviors. However, perceptions of the source or sponsor of the advertisement may influence consumers' attitudes and therefore intentions along the way. This study seeks to examine the role of fear versus scare appeals and perceived social responsibility of the sponsor in social marketing campaign messages to motivate consumers to seek further information and medical care; in this study specifically for Type II diabetes.
\end{abstract}

\section{References available upon request.}

\author{
C.D. Hopkins \\ Clemson University, Clemson, SC, USA \\ e-mail: chopkin@clemon.edu \\ K.J. Shanahan $(\varangle)$ \\ Mississippi State University, Starkville, MS, USA \\ e-mail: kshanahan@business.msstate.edu \\ K. Hood \\ Eastern Kentucky University, Richmond, KY, USA \\ e-mail: Karen.hood@eku.edu
}

\author{
A. White \\ Loyola University New Orleans, New Orleans, LA, USA \\ e-mail: allynwhite@ business.loyno.edu
}

